\title{
AN ULTRA LOW NOISE AC BEAM TRANSFORMER FOR DECELERATION AND DIAGNOSTICS OF LOW INTENSITY BEAMS.
}

\author{
C. Gonzalez and F. Pedersen ${ }^{\#}$, CERN, Geneva, Switzerland
}

\section{Abstract}

The design of a broad band ultra-low noise ferrite loaded AC beam transformer is presented. It is designed for use in the CERN Antiproton Decelerator [1] (AD), where beams of a few $10^{7}$ charges must be decelerated from 3.5 $\mathrm{GeV} / \mathrm{c}$ to $100 \mathrm{MeV} / \mathrm{c}$. It is used in the RF beam-phase loop, and for intensity and bunch shape measurements during deceleration. When the beam is debunched for cooling on magnetic flat tops, the pick-up is used for measurements of intensity and momentum distribution by means of longitudinal Schottky scans. When used as Schottky pick-up, the signal to noise ratio should be better by about $40 \mathrm{~dB}$ than the existing stripline based longitudinal Schottky pick-up. The integrated design of pick-up and associated low-noise amplifier is presented. The achieved noise performance of a few $\mathrm{pA} / \sqrt{\mathrm{Hz}}$ from 1 to $3 \mathrm{MHz}$ is obtained by attaching a low-noise, highimpedance silicon JFET (junction field effect transistor) amplifier to a high-Q resonant ferrite loaded cavity, and then eliminating the resonant response by low-noise RF feedback such that broad band response over almost 2 decades of frequency $(0.3-15 \mathrm{MHz})$ is obtained. The longitudinal coupling impedance is close to $10 \Omega$ in this frequency range, and the equivalent noise temperature of this resistance is about $0.4{ }^{\circ} \mathrm{K}$ mid-band although all components operate at ambient temperature. Finally the application of a similar design for single-pass bunch intensity measurements is discussed.

\section{INTRODUCTION}

The DC beam currents in the CERN AD (typically 0.2 to $15 \mu \mathrm{A}$ with antiprotons) are too low to be measured with a DC beam transformer. Beam intensity is therefore measured by RF beam current measurements when the beam is bunched and longitudinal Schottky scans when the beam is debunched. The range of RF frequencies is 0.17 to $1.6 \mathrm{MHz}$, so to measure these quantities as well as bunch lengths an ultra-low noise AC beam transformer with a bandwidth from 0.1 to $15 \mathrm{MHz}$ is required.

\section{BEAM TRANSFORMER AND HEAD AMPLIFIER}

The AC beam transformer consists of a ferrite loaded cavity with a ceramic gap in the beam pipe, a secondary winding of one additional turn (the cavity enclosure

\# Email: Flemming.Pedersen@cern.ch forms the first turn), and a low noise head amplifier with feedback connected to the secondary winding and mounted close to the cavity, Figure 1.

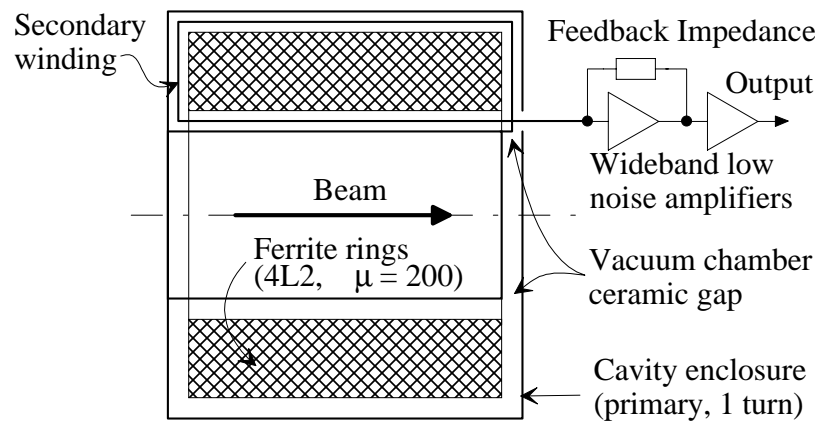

Figure 1. Ferrite loaded beam transformer and amplifier

A noise free amplifier cannot reduce noise already introduced by the pick-up itself, so the pick-up is made resonant with high $Q$ to reduce this noise. The whole device is doubly shielded: an outer cavity $(7 \mathrm{~mm}$ copper walls) surrounds the head amplifier and the inner cavity (also $7 \mathrm{~mm}$ copper), which contains the ferrite, the ceramic gap and the secondary winding and is assembled by e-beam welding to avoid RF contacts.

\subsection{Transformer and Amplifier Noise Sources}

It is easier and more transparent to characterise the noise properties of the amplifier [2] in terms of equivalent current and voltage noise sources and their dependence on frequency, device type and bias rather than noise figure which is often confusing.

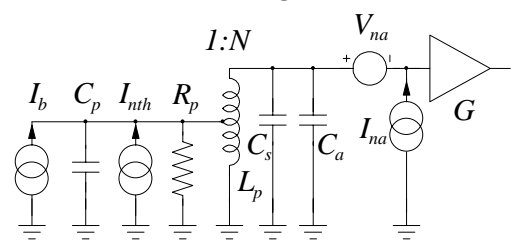

Figure 2: Equivalent circuit and noise sources

The equivalent circuit for the resonant step-up transformer and its head amplifier is shown on Figure 2.

Here $N$ is transformer step-up ratio, $I_{b}$ the desired signal, $V_{n a}[\mathrm{~V} / \sqrt{\mathrm{Hz}}]$ and $I_{n a}[\mathrm{~A} / \sqrt{\mathrm{Hz}}]$ amplifier voltage and current noise, $C_{p}$ and $C_{s}$ primary and secondary capacities of the transformer, $C_{a}$ amplifier input capacity, $L_{p}$ primary inductance, and $G$ amplifier gain. The Johnson noise $I_{n t h}$ of the shunt impedance $R_{p}(\sim 9 \mathrm{k} \Omega)$ is:

$I_{n t h}=\sqrt{4 k T / R_{p}}$ 
where $k\left[\mathrm{~J} /{ }^{\circ} \mathrm{K}\right]$ is Boltzman's constant and $T\left[{ }^{\circ} \mathrm{K}\right]$ the absolute temperature.

The secondary circuit quantities can all be transformed to the primary circuit, Figure 3. Increasing $N$ lowers voltage noise, but increases current noise and capacity.

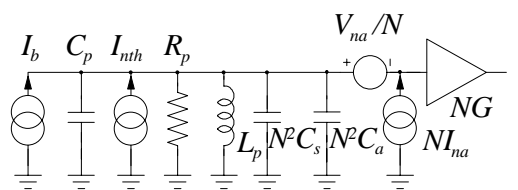

Figure 3: Noise sources transformed to primary

The amplifier voltage noise $V_{n d} / N$ can be transformed into an equivalent current noise $I_{v n a}$ by the relation:

$I_{\text {vna }}=V_{n a} /\left(N Z_{p u}\right)$

where $Z_{p u}$ is the primary pick-up impedance. If the 3 noise currents are uncorrelated (not quite true for $I_{a}$ and $V_{n a}$ ), they add as sum of the squares, and the total equivalent, input-noise current $I_{n, \text { in }}$ becomes:

$$
I_{n, i n}=\sqrt{I_{n t h}^{2}+\left(N I_{a}\right)^{2}+\left(V_{n a} / N Z_{p u}\right)^{2}}
$$

There is an optimum value of $N$ at a given frequency as amplifier current noise can be traded for voltage noise. If the amplifier noise is small, the total noise is dominated by the shunt impedance noise.

\subsection{Amplifier Design}

While bipolar transistors may have low voltage noise, the current noise at low frequencies $f \ll f_{T}$ is much larger than for FETs (field effect transistors) due to the electronic shot (Schottky) noise $I_{S}$ :

$I_{s}=\sqrt{2 q I_{\text {base }}}[\mathrm{A} / \sqrt{\mathrm{Hz}}]$

where $q$ is the elementary charge and $I_{\text {base }}$ the DC base bias current. A FET has an input voltage noise [3] closely related to the Johnson noise of the conducting channel:

$V_{n}=\sqrt{8 k T / 3 g_{m}} \quad[\mathrm{~V} / \sqrt{\mathrm{Hz}}]$

where $g_{m}$ is the transconductance $[\mathrm{A} / \mathrm{V}]$, and an input current noise which is proportional to frequency:

$I_{n}=\omega C_{c} V_{n}[\mathrm{~A} / \sqrt{\mathrm{Hz}}]$

where $C_{c}$ is the coupling capacitance, which is about $2 / 3$ of the total gate to channel capacity $C_{g s}$. Due to the very low gate bias current, the Schottky noise can usually be neglected. It is always possible to lower the noise voltage by connecting several transistors in parallel (square root dependence), but this will of course increase the noise current in the same ratio. This is similar to the noise matching obtained with the step-up transformer discussed in the previous subsection. The noise quality (lowest current noise for a given voltage noise) is therefore given by the ratio $g_{m} / C_{g s}$, which is also the high frequency figure of merit $f_{T}$. At frequencies below a certain corner frequency, all devices exhibit $1 / f$ noise or flicker noise, where noise spectral densities higher than $V_{n}$ given above (5) are observed.

The different types of FET transistors which may be considered [4] are: i) GaAs (Gallium Arsenide) MESFET: very high $f_{T}$ (typically $15-25 \mathrm{GHz}$ ), but also high $1 / f$ corner frequency: > $10 \mathrm{MHz}$ typically, ii) $\mathrm{Si}$ (silicon) dual gate MOSFET: lower $f_{T}$ (typically. $5 \mathrm{GHz}$ ), but somewhat better $1 / f$ corner frequency: $1-5 \mathrm{MHz}$, iii) $\mathrm{Si}$ JFET: still lower $f_{T}$ (typically $2 \mathrm{GHz}$ ), but much lower 1/f corner frequency: typically $10 \mathrm{~Hz}$ for Philips BF861C.

For the frequency range of interest ( 0.1 to $20 \mathrm{MHz}$ ), $\mathrm{Si}$ JFET's will be the best choice due to the low $1 / f$ corner frequency. Although monolithic amplifiers with JFET inputs are available, none of them have sufficiently low voltage noise and propagation delay $\left(t_{d} \leq 5 \mathrm{~ns}\right.$ required for feedback stability) and an amplifier has therefore been designed with discrete SMD components.

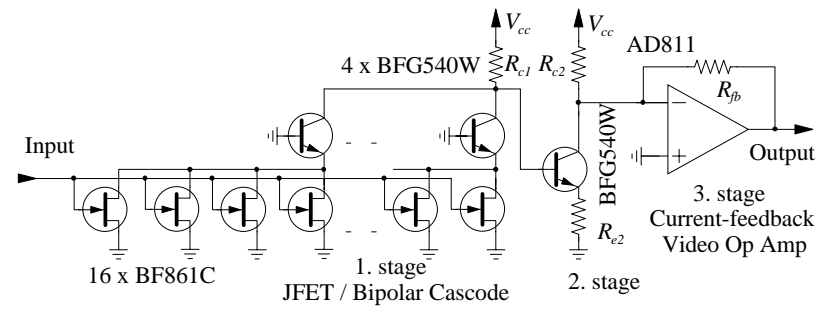

Figure 4: Low noise wide band amplifier

The conceptual design (bias details omitted) of the low noise, wide bandwidth feedback amplifier with an overall gain of 300 and a propagation delay of $5 \mathrm{~ns}$ (bandwidth $75 \mathrm{MHz}$ ) is shown on Figure 4. The input stage with a gain of 11 consists of 16 parallel Si JFETs with each group of 4 forming a cascode with a bipolar transistor with high $f_{T}(8 \mathrm{GHz})$. The JFETs are operating near their maximum current at $10 \mathrm{~mA}$ (for high $g_{m}$ and low $V_{n}$ ) and the bipolar transistors near their optimum current of 40 $\mathrm{mA}$. This results in a low input voltage noise (theory $0.22 \mathrm{nV} / \sqrt{\mathrm{Hz}}$, achieved $0.28 \mathrm{nV} / \sqrt{\mathrm{Hz}}$ ), and a very small propagation delay: $<1 \mathrm{~ns}$.

The output stage is a current mode feedback op-amp with very high slew rate $(2.5 \mathrm{kV} / \mu \mathrm{s})$ and voltage swing $\left(20 \mathrm{~V}_{\mathrm{pp}}\right)$ capable of driving the low impedance feedback circuitry. The bipolar second stage provides the extra gain to prevent the rather noisy output stage from contributing to the noise. For this amplifier design, the equivalent, input-noise current sources are shown on Figure 5. The cavity shunt impedance noise $(\sim 1.5 \mathrm{pA} / \sqrt{\mathrm{Hz}})$ dominates from 1.5 to $3.5 \mathrm{MHz}$, and corresponds to a noise temperature of $0.4^{\circ} \mathrm{K}$ of a $10 \Omega$ resistor. The longitudinal Schottky noise current densities for the AD are typically 4 to $500 \mathrm{pA} / \sqrt{\mathrm{Hz}}$ around $1.6 \mathrm{MHz}$ and lower at higher frequencies as the width of the Schottky bands are 
proportional to the harmonic while the total power in each Schottky band is constant.

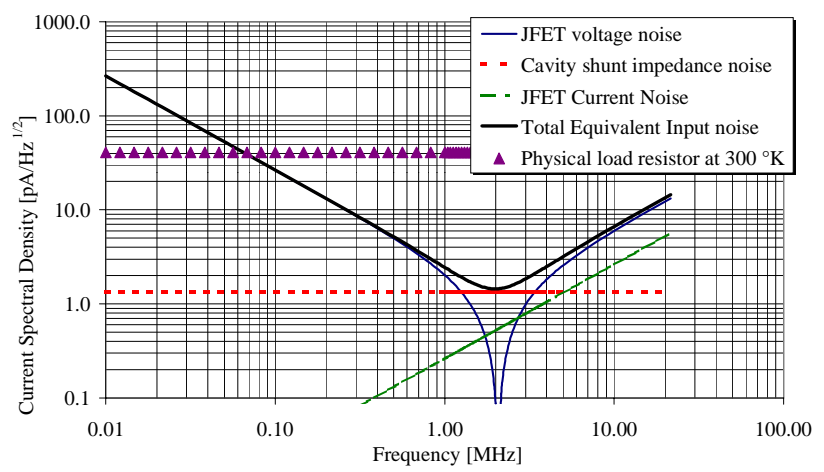

Figure 5: Equivalent, input-noise current sources (at gap)

\subsection{Feedback Resistor and Circuits}

Although the resonant high Q transformer with amplifier has a very good signal to noise ratio, the response is highly resonant $\left(Q \sim 120, f_{r} \sim 2 \mathrm{MHz}\right)$ and the amplifier will saturate for very small currents near the resonance. The response can be made broad band if a current feedback resistor is introduced around the amplifier, Figure 6. This converts the amplifier input impedance into a low resistive impedance $R_{f b} / G \sim 40 \Omega$, or $R_{f b} /\left(G N^{2}\right)$ $\sim 10 \Omega$ referred to primary and the response becomes broad band with $3 \mathrm{~dB}$ frequencies of 0.3 and $15 \mathrm{MHz}$ and a loop gain at resonance about $60 \mathrm{~dB}$.

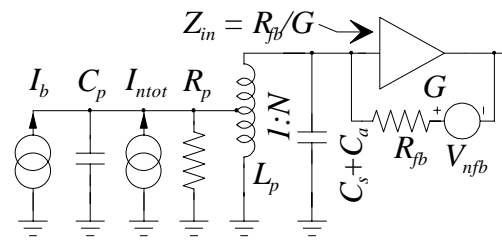

Figure 6: Equivalent circuit with current feedback

However, unless the feedback resistor and the gain are made very large $\left(R_{f b}>0.4 \mathrm{M} \Omega, \mathrm{G}>10^{\prime} 000\right)$, the noise of the feedback resistor will contribute significantly to (or even dominate) the noise. A large feedback resistor is difficult to implement at high frequencies, the amplifier will still saturate at very low currents, and large gain with short delay is difficult.

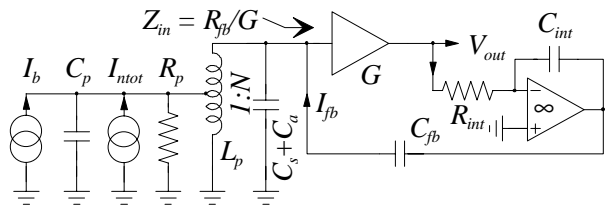

Figure 7: Low noise feedback 'resistor'

By passing the feedback signal through an integrator and injecting the feedback current through a small capacitor the equivalent feedback transfer function is still that of a resistor, Figure 7. For $R_{i n t}$ small and $C_{i n t} \gg C_{f b}$, the effective noise temperature $T_{\text {noise }}$ of the feedback resistor is low: $15^{\circ} \mathrm{K}$ for $R_{f b}=12 \mathrm{k} \Omega$, or a current noise corresponding to a $240 \mathrm{k} \Omega$ resistor. The dynamic range is much increased (saturation for $I_{b}=1.6 \mathrm{~mA}$ ) with only a very small increase in noise level.

\subsection{Low Frequency Version}

The low frequency response cut-off frequency $(0.3 \mathrm{MHz})$ can be lowered by an appropriate integrating correcting network in the feedback amplifier, but the low frequency noise current is unchanged: limited by inductance $L_{p}=6 \mu \mathrm{H}$ and noise voltage $V_{n} / N=0.14 \mathrm{nV} / \sqrt{\mathrm{Hz}}$. To decrease the low frequency cut-off and at the same time lower the noise by $10-20 \mathrm{~dB}$ at $400 \mathrm{kHz}$ and below, a low frequency cavity with higher inductance (4A15 ferrite, $\mu=1200, L_{p}=40 \mu \mathrm{H}$ ) and with a step-up ratio of $N=4$ (which halves the noise voltage to $V_{n} / N=0.07 \mathrm{nV} / \sqrt{\mathrm{Hz}}$ ) has been built. It is installed in the $\mathrm{AD}$ ring adjacent to the high frequency device. Its response bandwidth is from $40 \mathrm{kHz}$ to $3 \mathrm{MHz}$. The signals from the two devices are then combined to a single broadband signal with a crossover frequency (1 MHz) chosen for minimum noise.

\section{LOW NOISE BEAM TRANSFORMER FOR BEAM TRANSFER LINE}

To measure the charge of the extracted low intensity antiproton bunch (about $10^{7}$ charges, length about $300 \mathrm{~ns}$ ), an additional transformer (low frequency version) will be installed in the extraction line. The extracted charge is measured by integrating the current signal during $t_{i}=1 \mu \mathrm{s}$. A bandwidth of $3 \mathrm{kHz}(18 \mathrm{kHz}$ with response shaping) to $3 \mathrm{MHz}$ is needed for less than $1 \%$ error due to the response limitations. The mean square error of the charge signal $q$ due to noise is related to the (double-sided) power spectral density of the current noise $G_{i}(f)\left[\mathrm{A}^{2} / \mathrm{Hz}\right]$ :

$\overline{q^{2}}=t_{i}^{2} \int_{0}^{\infty} 2 G_{i}(f) \frac{\sin ^{2}\left(\omega t_{i} / 2\right)}{\left(\omega t_{i} / 2\right)^{2}} d f$

which results in an expected RMS fluctuation of $1.9 \times 10^{4}$ charges ( 4 sigma is $7.6 \times 10^{4}$ charges).

\section{REFERENCES}

[1] S. Baird and 29 co-authors, "The Antiproton Decelerator: AD". Proc. 1997 Particle Accelerator Conference, Vancouver, Canada (1997) [2] Y. Netzer, "The Design of Low-Noise Amplifiers", Proc. of the IEEE, Vol. 69, 728 (1981)

[3] A. van der Ziel, "Noise: Sources, Characteristics, Measurements" (Prentice Hall, Englewood Cliffs, NJ, 1970)

[4] F. Celani et al. "Behaviour and comparison of RF devices Si JFET, dual gate MOSFET and GaAs MESFET...", Proc. of the Symposium on Low Temperature Electronics and High Temperature Superconductors, Honolulu, HI, USA, p.476-88 (1987) 